\title{
Diagnosis and treatment of alcoholic hepatitis
}

\author{
R Parker, $^{1,2}$ C A McCune ${ }^{3}$
}

\begin{abstract}
- Additional material is published online only. To view please visit the journal online (http://dx.doi.org/10.1136/ flgastro-2013-100373).

${ }^{1}$ NIHR Centre for Liver Research, University of Birmingham, Birmingham, UK

${ }^{2}$ Liver Unit, University Hospitals Birmingham NHS Foundation Trust, Birmingham, UK ${ }^{3}$ Department of Liver Medicine, University Hospitals Bristol NHS Foundation Trust, Bristol, UK
\end{abstract}

\section{Correspondence to}

Dr Richard Parker, NIHR Centre for Liver Research, University of Birmingham, Birmingham B15 2TT, UK; richardparker@nhs.net

Received 5 August 2013 Revised 30 September 2013 Accepted 2 October 2013 Published Online First 1 November 2013
To cite: Parker $R$, McCune CA. Frontline Gastroenterology 2014;5:123-129.

\section{ABSTRACT}

Alcoholic liver disease (ALD) is increasing in incidence in the UK. It is the commonest cause of liver-related deaths, predominantly in people below the age of 60 . Alcoholic hepatitis $(A H)$ is an acute form of ALD with high mortality when severe. Jaundice and coagulopathy are clinical hallmarks of severe $\mathrm{AH}$. Histology findings are characterised by parenchymal inflammation and hepatocellular damage although biopsy is only required when diagnostic uncertainty exists; clinical findings are usually sufficient for accurate diagnosis. Patients with $\mathrm{AH}$ should be stratified as non-severe or severe using non-invasive scoring systems such as the discriminant function or the Glasgow Alcoholic Hepatitis Score. In patients with non-severe $\mathrm{AH}$, abstinence is the mainstay of treatment, and it is important that steps are taken to help patients stop drinking. Severe $\mathrm{AH}$ requires specialist treatment. Consensus guidelines recommend the use of prednisolone although this remains subject to clinical trials. Pentoxifylline may have a survival benefit if corticosteroids are contraindicated. Nutritional support and $\mathrm{N}$-acetylcysteine should be considered for use in conjunction with corticosteroids although evidence of benefit is not conclusive. Patients with severe disease who do not respond to therapy within a week have a very poor outcome. Recent data have shown a survival benefit of liver transplantation in this group although this remains experimental at present. Current and future research should focus on targeted therapies for severe $\mathrm{AH}$ and those who fail first-line treatment.

\section{INTRODUCTION}

Alcoholic liver disease (ALD) is a spectrumencompassing steatosis, steatohepatitis, cirrhosis and hepatocellular carcinoma. The incidence of ALD is increasing in the UK, and is the commonest cause of cirrhosis ${ }^{1}$ and liver-related deaths, responsible for nearly 4000 deaths/year in England. Deaths from ALD occur predominantly in a relatively young population, below the age of $60 .^{2}$ Alcoholic hepatitis (AH) is a florid manifestation of ALD characterised by parenchymal inflammation and hepatocellular damage, ${ }^{3}$ in the presence of recent hazardous alcohol intake. Non-severe AH may be asymptomatic and generally requires no treatment other than abstinence, but increases the risk of progression to cirrhosis and medium-term/long-term mortality. Severe AH presents with jaundice, malaise, fever and signs of liver failure and has a poor short-term prognosis. ${ }^{4}$ Severe $\mathrm{AH}$ usually occurs in patients with underlying cirrhosis, but may occur in individuals without significant fibrosis.

The American Society for the Study of Liver Disease (AASLD) ${ }^{5}$ and the European Association for the Study of the Liver (EASL) ${ }^{3}$ have recently published comprehensive guidelines for management of ALD. Additionally, the National Institute for Health and Care Excellence (NICE) in the UK has published guidance on a wide range of alcohol-related health disorders including $\mathrm{AH}^{6}{ }^{6}$ This topic has relevance to the 2010 gastroenterology curriculum, specifically hepatology competencies $2 \mathrm{e}$ (clinical evaluation of liver disease, jaundice, specific diseases: alcohol and the liver) and advanced hepatology 3a (liver transplantation).

\section{ALCOHOLIC HEPATITIS}

Alcohol is normally predominantly metabolised to acetaldehyde by alcohol dehydrogenase in the liver. Products of alcohol metabolism can replace fatty acids in the Krebs cycle and promote lipogenesis, leading to hepatic steatosis. ${ }^{7}$ In chronic alcohol excess the microsomal ethanol-oxidising system and peroxisomal catalase are induced to metabolise alcohol. Acetaldehyde is highly toxic and 
forms adducts with proteins, lipids and DNA, impairing their function and promoting DNA damage. Protein adducts are also immunogenic, contributing to the inflammatory infiltrate seen in $\mathrm{AH}$. Inducible pathways of alcohol metabolism lead to the production of damaging free radicals. Activation of macrophages and Kupffer cells (liver-resident macrophages) leads to secretion of pro-inflammatory cytokines, such as IL-1, IL-6 and Tumour Necrosis Factor $\alpha(\mathrm{TNF} \alpha){ }^{8}$ $\mathrm{TNF} \alpha$ is significantly elevated in severe $\mathrm{AH}$, and can mediate hepatocyte death directly as well as contributing to immune cell infiltration. ${ }^{9}$ Neutrophils migrate into the parenchyma leading to hepatocyte necrosis. The degree of neutrophil infiltration correlates with severity of AH. T-lymphocytes are also recruited to the site of inflammation and mediate hepatocyte death through necrosis or apoptosis. Necrosis is characterised by hepatocyte ballooning, a typical histological finding in AH. Additionally, alcohol reduces gut wall integrity, allowing gut-derived lipopolysaccharide to cross into the portal venous system activating cells of the innate immune system and contributing to hepatic inflammation.

$\mathrm{AH}$ is increasing in incidence. The prevalence of $\mathrm{AH}$ is unknown, as mild disease may be present in up to $10 \%$ of otherwise healthy individuals who drink hazardously, and can persist for months in hazardous drinkers who have become abstinent. Progression of $\mathrm{AH}$ is strongly linked to continuing alcohol consumption: histological improvement is more likely with abstinence or controlled intake. In severe $\mathrm{AH}$, death usually occurs from liver failure, gastrointestinal bleeding or infection. ${ }^{10}$

\section{DIAGNOSIS AND ASSESSMENT OF ACUTE AH}

The principal clinical feature of $\mathrm{AH}$ is jaundice, which may be accompanied by a plethora of symptoms and signs. Malaise, confusion, abdominal pain (from capsular distension), anorexia may be present. Signs of chronic liver disease are frequently present as up to $80 \%$ of patients with $\mathrm{AH}$ will have underlying cirrhosis. Typical blood tests abnormalities are summarised in table 1, where raised bilirubin and coagulopathy are hallmarks of severe AH. Aspartate aminotransferase (AST) may be moderately raised, usually higher than alanine transferase (ALT) concentration. ${ }^{11}$ If significantly raised (ALT $>300)$, then an alternative diagnosis such as drugs, viral or ischaemia should be sought. Neutrophilia may be evident in the absence of infection. Impaired renal function is associated with a poor prognosis. ${ }^{12}$ Other causes of liver disease should be screened for as ALD may coexist with other pathology.

In patients with $\mathrm{AH}$, alcohol excess will usually have been present for years but may be underreported. Patients should be screened for alcohol dependence using a validated screening tool such as the AUDIT questionnaire or an abbreviated form, for example, AUDIT-C ${ }^{13}$ or FAST. ${ }^{14}$ If screening suggests
Table 1 Diagnostic features of alcoholic hepatitis

\begin{tabular}{ll}
\hline Biochemical/haematological & Histological \\
\hline Hyperbilirubinaemia $(>80 \mu \mathrm{mol} / \mathrm{L}$ ) & Hepatocyte ballooning \\
Raised AST (but less than $300 \mathrm{IU} / \mathrm{mL}$ ) & Hepatocyte necrosis \\
AST : ALT ratio $>2$ & Mallory-Denk bodies (cytoplasmic \\
& accumulations) \\
Prolonged prothrombin time & Fibrosis; often pericellular \\
Neutrophilia & Steatosis \\
& Giant mitochondria \\
& Inflammatory infiltrate \\
& Cholestasis \\
& Bile duct proliferation? \\
\hline
\end{tabular}

ALT, alanine transferase; AST, aspartate aminotransferase

alcohol dependence is present (AUDIT score $>20$ or AUDIT-C positive) referral for specialist assessment is recommended. ${ }^{15}$ Collateral history should be sought if there is clinical suspicion of ALD. Detailed assessment by addiction specialists may help to identify and clarify alcohol use. ${ }^{16}$ Laboratory tests such as carbohydrate deficient transferrin and mitochondrial aspartate aminotransferase can detect recent alcohol consumption but are not particularly accurate, and their utility in the context of severe liver dysfunction is unknown.

Histological features of $\mathrm{AH}$ are shown in table 1. Liver biopsy can clarify cases where diagnostic uncertainty exists, although its role remains controversial. A decision regarding biopsy and subsequent treatment should be made by a clinician with hepatology expertise. Complications of biopsy are rare, although more common in patients with clotting disorders and cirrhosis. Clotting derangement and ascites due to $\mathrm{AH}$ often necessitates trans-jugular biopsy. If a biopsy is felt necessary, the result should be available to the clinical team within $48 \mathrm{~h}$ to avoid unnecessary delays in treatment. Prompt transfer to a specialist centre may be required if necessary facilities and expertise are not available locally. Current consensus guidelines broadly agree that biopsy should be considered in severe cases of $\mathrm{AH}$ that require treatment. ${ }^{3} 56$ Current trials may bring some clarity to this area. Fibroscan (non-invasive elastography), is unreliable in severe $\mathrm{AH}$ as acute inflammation causes an apparent increase in liver stiffness. ${ }^{17}$-at least 3 months of abstinence is recommended before Fibroscan might be reliably used.

\section{Assessing severity}

A number of scoring systems have been used to gauge severity of $\mathrm{AH}$ (table 2. The discriminant function (DF) (widely referred to as the Maddrey score) was described when bilirubin and prothrombin time were found to predict death in a trial of prednisolone in 55 patients with $\mathrm{AH}$. The score was subsequently modified to its present form where a score of 32 or above indicates severe disease, associated with 28-day mortality of around $35 \%$ if untreated and $20 \%$ with 


\begin{tabular}{|c|c|c|c|c|c|c|c|c|c|}
\hline \multirow[b]{2}{*}{ Score } & \multirow[b]{2}{*}{ Formula } & & & & \multicolumn{3}{|c|}{$\begin{array}{l}\text { Cut-offs for non-severe/ } \\
\text { severe disease }\end{array}$} & \multicolumn{2}{|c|}{ Approximate 28 -day survival if severe } \\
\hline & & & & & Low & High & & Without treatment & With treatment \\
\hline$\overline{\mathrm{DF}}$ & \multicolumn{4}{|c|}{4.5 (PT-control PT)+(bilirubin/17) } & $<32$ & $32+$ & & $65 \%$ & $80 \%$ \\
\hline GAHS & $\begin{array}{l}\text { Age } \\
\text { WCC } \\
\text { Urea } \\
\text { INR } \\
\text { Bilirubin }\end{array}$ & $\begin{array}{l}1 \\
<50 \\
<1.5 \\
<5 \\
<1.5 \\
<125\end{array}$ & $\begin{array}{l}2 \\
50+ \\
1.5+ \\
5+ \\
1.5-2 \\
125-250\end{array}$ & $\begin{array}{l}2+ \\
>250\end{array}$ & $<9$ & $9+$ & & $50 \%$ & $78 \%$ \\
\hline$A B I C$ & \multicolumn{4}{|c|}{$\begin{array}{l}\text { (age years } \times 0.01)+(\text { bilirubin } \mathrm{mg} / \mathrm{dL} \times 0.8) \\
+(\text { creatinine } \mathrm{mg} / \mathrm{dL} \times 0.3)+(\mathrm{INR} \times 0.8)\end{array}$} & $<6.71$ & $6.71-8.9$ & $>8.9$ & NA & NA (6 months survival $0 \%$ ) \\
\hline
\end{tabular}

For GAHS, each variable scores a defined number of points which add up to the final score.

ABIC, Age, Bilirubin INR and Creatinine; GAHS, Glasgow Alcoholic Hepatitis Score; PT, prothrombin time.

treatment. ${ }^{4}$ The Glasgow Alcoholic Hepatitis Score (GAHS) uses a scoring system including 5 variables. Severe disease is defined by a score of 9 or above, associated with $54 \%$ mortality at 28 days compared to $13 \%$ if the GAHS is below 9. The ABIC (Age, Bilirubin, international normalised ratio (INR) and Creatinine) score stratifies patients with acute $\mathrm{AH}$ into those at low, high and indeterminate risk of death at 90 days with $0,30 \%$ and $75 \%$ mortality, respectively. Subsequent studies comparing these scores have found little difference in their ability to predict outcome, but the GAHS is superior in identifying patients who will benefit from coroticosteriods. ${ }^{18}$ Scoring systems not designed specifically for use in $\mathrm{AH}$, such as model for end-stage liver disease (MELD) or Child-PughTurncott, can also predict survival in AH. These scores are designed to assess severity; they are not diagnostic tools and should not be regarded as such. The Lille model (discussed below) is designed to assess response to corticosteroid treatment and should not be used to assess severity at admission.

\section{TREATMENT OF AH}

In patients with non-severe $\mathrm{AH}$, abstinence is the mainstay of treatment, ${ }^{19}$ severe $\mathrm{AH}$ requires specific treatment. $^{4}$ Patients with severe $\mathrm{AH}$ are often extremely sick and require early specialist input. Patients with severe alcohol-related hepatitis should be treated by medical and nursing teams experienced in the management of decompensated liver disease with additional input from alcohol liaison staff, nutritionists and intensivists where appropriate. Early specialist review is of particular importance in deciding whether a patient receives pharmacological treatment (see below) or undergoes liver biopsy. An outline of diagnosis and management is given in figure 1.

\section{General measures}

Management of alcohol withdrawal/dependence

Many patients with $\mathrm{AH}$ will be physically dependent on alcohol, although this is not universal. Long-term abstinence improves prognosis in all forms of ALD including AH. Alcohol withdrawal should be managed with benzodiazepines to alleviate symptoms of withdrawal and progression to delirium tremens (DTs). Oxazepam may be preferred in patients with severe liver dysfunction as it has a shorter half-life and, thus, may be less likely to accumulate, although it requires more frequent assessment. High dependency or intensive care may be required to safely manage severe withdrawal/ DTs. Patients identified as high risk of developing Wernicke-Korsakoff syndrome (WKS) should be prophylactically treated with parenteral thiamine treatment. A high level of suspicion for the possibility of Wernicke's encephalopathy is required, particularly if the person is intoxicated. If there is uncertainty or if signs of WKS are present, then treatment with high-dose parenteral thiamine should be given for a minimum of 5 days, unless Wernicke's encephalopathy is excluded. Oral thiamine treatment should follow parenteral therapy. ${ }^{6}$ Interventions to achieve long-term abstinence are mainly based around psychological interventions, and addiction specialists should be routinely involved in the care of patients with $\mathrm{AH}$.

\section{Treatment of sepsis}

Between 25\% and 50\% of patients with $\mathrm{AH}$ will have superadded sepsis which is often fatal, ${ }^{20}$ thus, great care should be taken with venous cannulae, urinary catheters and the like, to prevent infections in patients with severe AH. Sepsis may be difficult to rule out, as many patients with severe $\mathrm{AH}(>50 \%)$ will have Systemic Inflammatory Response Syndrome (SIRS) ${ }^{21}$ and a raised neutrophil count. ${ }^{22}$ Patients should have a full sepsis screen (blood culture, urine culture, culture of ascitic fluid and chest radiograph) on admission and regularly thereafter according to the patient's clinical condition. There should be a low threshold for treatment with antibiotics, although there is no evidence to support the use of prophylactic antibiotics in the absence of proven infection. Aminoglycoside antibiotics, such as gentamicin and vancomycin, should be avoided as they can impair 


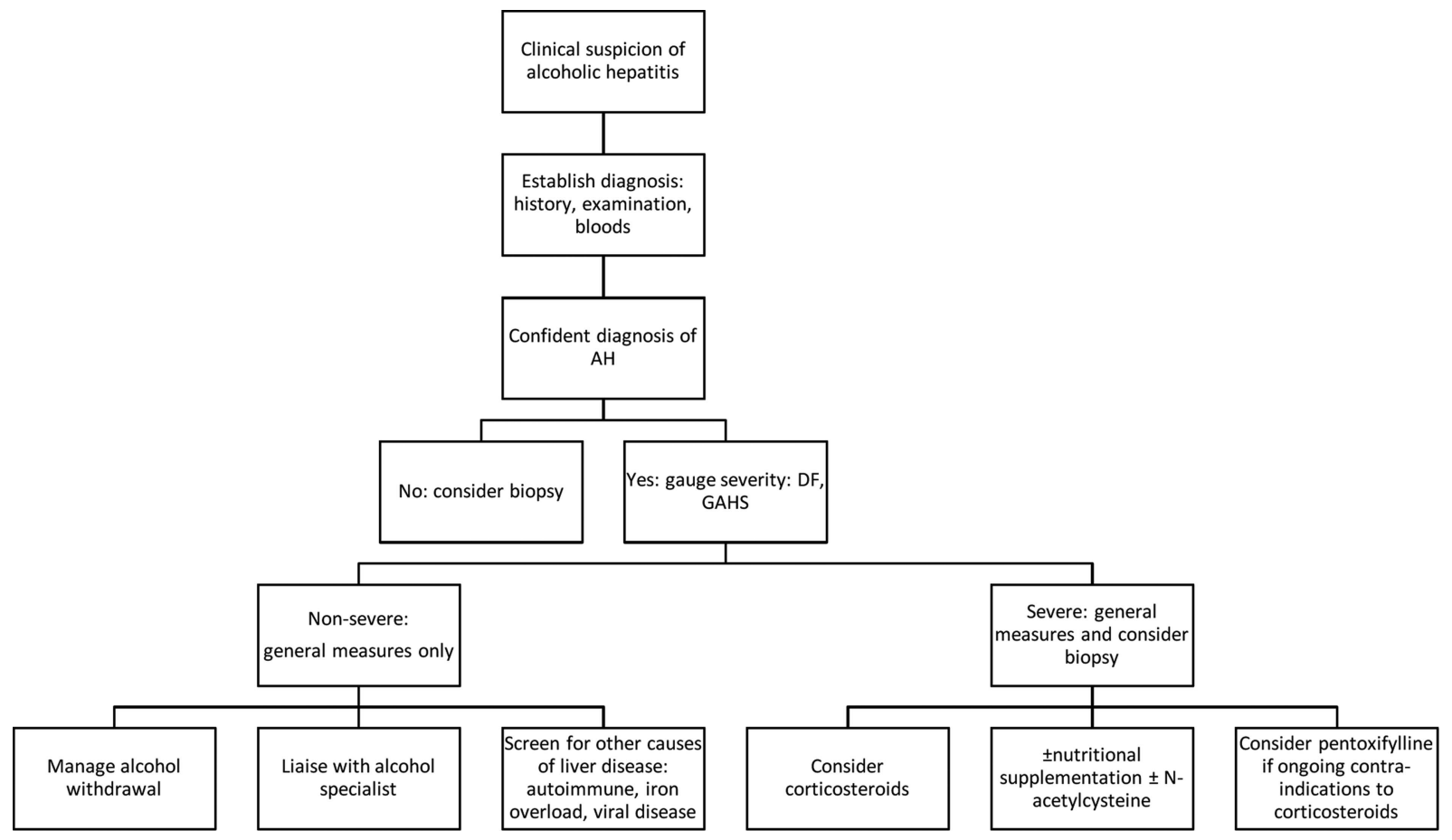

Figure 1 Suggested algorithm to assess and treat alcoholic hepatitis ( $\mathrm{AH}$; adapted from European Association for the Study of the Liver (EASL) guidelines).

already vulnerable renal function-liaison with microbiology is advised. Patients with severe $\mathrm{AH}$ are immunocompromised, and antifungal agents may be required in those who fail to respond to first-line broad-spectrum antibiotics.

Treatment of complications of cirrhosis

AH may cause decompensation of chronic liver disease. Variceal bleeding should be treated as per usual protocols with an emphasis on prompt circulatory support and control of bleeding. Sedation in patients with acute liver dysfunction should be undertaken with caution. Encephalopathic patients should be treated with bowel care and minimising medications that may precipitate encephalopathy. Care should be taken to rule out Wernicke's encephalopathy. Patients with $\mathrm{AH}$ and acute kidney injury (AKI) have a poorer prognosis $(67 \%$ mortality vs $7 \%$ in patients without AKI). ${ }^{12}$ Renal function needs to be very carefully monitored, and it is important that measures are taken to prevent the development of AKI, for example, avoidance of nephrotoxic drugs. Hepatorenal syndrome (HRS) is a frequent diagnosis in patients with $\mathrm{AH}$, but most cases of renal impairment are due to other causes. ${ }^{2}$ If HRS is present, standard measures, such as intravenous albumin and terlipressin are appropriate.

\section{Specific treatment}

\section{Corticosteroids}

Corticosteroids have been the subject of much research. Meta-analysis ${ }^{4}$ of five trials showed a survival benefit with corticosteroids in patients with a $\mathrm{DF}>32$. Pooled data in this meta-analysis showed survival of $79.97 \%( \pm 2.8 \%)$ in the corticosteroid group and $65.7 \%( \pm 3.4)$ in the placebo group $(\mathrm{p}=0.0005)$ at 28 days. This was confirmed by meta-analysis performed by NICE. ${ }^{6}$ Several different corticosteroids have been used in clinical trials; the most commonly used is prednisolone. Consensus guidelines recommend prednisolone at a dose of $40 \mathrm{mg} /$ day for 28 days. There are no data to describe whether complete cessation of prednisolone or a tapering dose is most appropriate after 28 days. Despite repeated meta-analyses (of heterogenous and often underpowered trials) there remains reluctance among some physicians to use them for treatment of severe $\mathrm{AH}$, and they remain the subject of clinical trials.

Response to corticosteroid therapy, indicated by improving biochemistry, is an important prognostic sign. A 25\% reduction in bilirubin level after 7-9 days of treatment is a simple and accurate method to assess response. The Lille model was derived in a group of highly selected patients with severe $\mathrm{AH}(\mathrm{mDF}>32$ or encephalopathy). In this more complex system (http:// www.lillemodel.com), a score higher than 0.45 is associated with a much worse 6 -month mortality $(25 \%$ vs $85 \%)$. EASL guidelines recommend treatment is stopped in non-responders. Rescue therapy with pentoxifylline has been shown to be ineffective. There is an urgent need for effective treatments in this group of patients.

Infections are not an absolute contraindication to corticosteroid therapy: treatment can be started once 
infection is resolving with similar outcomes to patients without infections. The recent meta-analysis by NICE showed no significant increase in risk of GI bleeding or infection with corticosteroid use ${ }^{6}$ although studies have generally excluded such high-risk patients. However, development of de novo sepsis once treatment with corticosteroids started reduces response to therapy and is associated with worse outcomes.

\section{Pentoxifylline}

TNF- $\alpha$ plays an important role in the pathogenesis of AH. Pentoxifylline is an anti-TNF $\alpha$ agent through inhibition of phosphodiesterase. The single good quality randomised controlled trial (RCT) of pentoxifylline showed improvement in in-hospital survival (24.5\% vs $46.1 \%)$ in severe $\mathrm{AH}$, thought to be due to a reduction in incidence of hepatorenal syndrome. Later trials comparing pentoxifylline to prednisolone have significant methodological flaws. Larger, wellperformed trials have not shown additional benefit of combination of pentoxifylline and prednisolone. Meta-analysis of published trials does not show a survival benefit of pentoxifylline as monotherapy or with corticosteroid. ${ }^{23}$

\section{Nutritional supplementation}

Poor nutritional status is common among patients with AH. Nutritional supplements (oral or NG) can maintain gut integrity and reduce translocation of gutderived bacteria and endotoxin load. Nutritional supplementation (ie, in addition to normal diet) with a $2000 \mathrm{kcal} /$ day diet, with or without branched amino acids, did not affect short-term mortality in AH. A later trial of enteral feeding of $2000 \mathrm{kcal} /$ day as sole nutritional intake in patients with severe $\mathrm{AH}$, was as effective as prednisolone therapy and oral diet, with regard to 28 -day mortality ( $31 \%$ vs $25 \%, \mathrm{p}=0.54$ ), and 1-year mortality $(37 \%$ vs $53 \%, p=0.23) .{ }^{24}$ Nasogastric (NG) feeding is not without risks, particularly in patients who may be or become encephalopathic.

\section{Antioxidants and $\mathrm{N}$-acetylcysteine}

Antioxidants are an attractive agent as they could counteract the oxidative stress shown to be an integral part of the pathogenesis of severe AH. Antioxidants alone are not effective in reducing short-term mortality in severe AH. ${ }^{25}{ }^{26}$ There are conflicting data as to whether antioxidants add benefit to prednisolone therapy. A small trial $(n=77)$ of 1 week of intravenous $\mathrm{N}$-acetylcysteine (NAC) and 6 months of oral antioxidants showed no additional benefit to prednisolone therapy. A study of 177 patients randomised to prednisolone with or without NAC showed reduced mortality with combination therapy at 28 days ( $8 \%$ vs $24 \%, p=0.006$ ), but the difference was not statistically significant at 6 months $(27 \%$ vs $38 \%, p=0.07) .{ }^{27}$
Intensive therapy unit therapy

A recent national confidential enquiry into patient outcome and death (NCEPOD) report found frequent reluctance to admit patients with $\mathrm{AH}$ to highdependency or intensive therapy units (ITU) despite clear indications for intensive therapy. ${ }^{2}$ This may be due to pejorative attitudes regarding patients with ALD rather than clinical assessment. Survival in ITU is related to the number of underlying organ failures rather than severity of liver disease, moreover, patients with $\mathrm{AH}$ may have better hospital survival than other causes of decompensation, and $\mathrm{AH}$ is a treatable condition. ITU admission may, therefore, be appropriate.

\section{Transplantation}

The presence of $\mathrm{AH}$ in explanted liver after transplant does not adversely affect outcome with regard to survival or relapse to drinking. A French trial in 26 highly selected patients (table 3) with severe $\mathrm{AH}$ who had failed medical therapy showed that liver transplantation improved 6-month survival in this group from $23 \%$ to $77 \% .^{28}$ It should be noted that while this survival is significantly improved in the context of $\mathrm{AH}$, it is much worse than might be expected for transplantation of chronic liver disease at 6 months. Three patients resumed drinking after transplantation. While donor organs are in short supply, this will remain a contentious issue.

\section{CURRENT TRIALS AND EXPERIMENTAL TREATMENTS}

Steroids or pentoxifylline in Alcoholic Hepatitis (STOPAH) is a large multicentre UK study open to recruitment at time of writing. ${ }^{29}$ This, the largest study conducted in $\mathrm{AH}$, aims to be definitive and to resolve the ongoing controversy around treatment. Eligibility criteria are based on clinical parameters, and thus, applicable to a UK patient cohort. Those presenting with sepsis, bleeding and renal failure are eligible for enrolment once these factors are controlled. 1200 patients will be randomised to placebo, prednisolone, pentoxifylline or combination therapy with prednisolone and pentoxifylline.

Steroid resistance can be identified at baseline and predicts outcome, thus improving efficacy of corticosteroid treatment may be of benefit. Agents, such as

Table 3 Criteria used to select patients with severe alcoholic hepatitis $(A H)$ for transplantation ${ }^{28}$

\begin{tabular}{|c|c|}
\hline Medical criteria & Psychosocial criteria \\
\hline First episode of $\mathrm{AH}$ & Commitment to abstinence \\
\hline $\begin{array}{l}\text { Treatment failure (Lille score }>0.45 \text {, } \\
\text { or rapid worsening of LFTs despite } \\
\text { therapy) }\end{array}$ & Supportive family \\
\hline No severe medical comorbidities & $\begin{array}{l}\text { Consensus of medical and } \\
\text { paramedical staff that } \\
\text { transplantation was justified }\end{array}$ \\
\hline
\end{tabular}


basiliximab $^{30}$ and theophylline ${ }^{31}$ have shown effect in vitro, their efficacy in vivo remains to be proven. Liver support devices appeared effective in pilot studies of patients with severe AH with regard to biochemical and haemodynamic effects. The ELAD device, based on human hepatocytes, is currently being trialled. ${ }^{32}$ Leukopheresis and infusions of autologous bone marrow-derived stem cells have failed to show efficacy. ${ }^{33} 34$

\section{CONCLUSION}

$\mathrm{AH}$ is a florid form of ALD that is increasing in incidence and is frequently fatal when severe. The management of ALD in the UK has recently been found to be poor, despite comprehensive consensus guidelines from specialist societies and NICE. Trainees in gastroenterology will inevitably be required to manage this disease and, as such, should be aware of the rationale, evidence and indications for the various treatment options. Unfortunately, many treatments for AH have been addressed in small underpowered trials, which limits the evidence base.

When $\mathrm{AH}$ is suspected, careful history taking and routine investigations are usually sufficient to establish a diagnosis. Biopsy should be reserved for cases of diagnostic uncertainty and should not delay starting treatment. Severity can be gauged using a variety of scoring systems to guide appropriate treatment aiding triage to specialist wards/centres. Consensus guidelines advise the use of corticosteroids in severe $\mathrm{AH}$. Use of $\mathrm{N}$-acetlycysteine as an adjunct to corticosteroids may be beneficial. Nutritional supplementation, orally or NG is important to address malnutrition which is frequent among those who abuse alcohol, NG feeding appeared as efficacious as corticosteroids in a small trial. Management of other complications of alcohol abuse should not be disregarded. The majority of patients with $\mathrm{AH}$ will have underlying cirrhosis, which may decompensate in the presence of AH. Escalation of treatment is appropriate when necessary, outcomes relate to the number of organ systems failing rather than the degree of liver failure. New treatments are urgently required to treat this devastating condition.

\section{Curriculum links}

2e hepatology basic principles: clinical evaluation of liver disease, jaundice

- 2e hepatology specific diseases: alcohol and the liver - 3a advanced hepatology: liver transplantation

Contributors RP had the initial idea for this article, and wrote the manuscript. CAM read and edited the draft manuscripts.

Funding RP is supported by Clinical Research Training Fellowship from the Medical Research Council grant number G1144008.
Competing interests None.

Provenance and peer review Not commissioned; externally peer reviewed.

\section{REFERENCES}

1 Leon DA, Mccambridge J. Liver cirrhosis mortality rates in britain from 1950 to 2002: an analysis of routine data. Lancet 2006;367:52-6.

2 National Institute for Healthcare and Clinical Excellence. Measuring the units: a review of patients who died with alcohol related liver disease. 2013.

3 EASL Clinical Practical Guidelines: management of alcoholic liver disease. J Hepatol 2012;57:399-420.

4 Mathurin P, O'grady J, Carithers RL, et al. Corticosteroids improve short-term survival in patients with severe alcoholic hepatitis: Meta-analysis of individual patient data. Gut 2011;60:255-60.

5 O'shea RS, Dasarathy S, Mccullough AJ. Alcoholic liver disease. Hepatology 2010;51:307-28.

6 National Institute for Healthcare and Clinical Excellence. Diagnosis and clinical management of alcohol related physical complications. 2010.

7 Purohit V, Gao B, Song B. Molecular mechanisms of alcoholic fatty liver. Alcohol Clin Exp Res 2009;33:191-205.

8 Gao B, Bataller R. Alcoholic liver disease: pathogenesis and new therapeutic targets. Gastroenterology 2011;141:1572-85.

9 Dhanda AD, Lee RWL, Collins PL, et al. Molecular targets in the treatment of alcoholic hepatitis. World J Gastroenterol 2012;18:5504.

$10 \mathrm{Yu} \mathrm{CH}, \mathrm{Xu} \mathrm{CF}, \mathrm{Ye} \mathrm{H}$, et al. Early mortality of alcoholic hepatitis: a review of data from placebo-controlled clinical trials. World J Gastroenterol 2010;16:2435.

11 Lucey MR, Mathurin P, Morgan TR. Alcoholic hepatitis. N Engl J Med 2009;360:2758-69.

12 Altamirano J, Fagundes C, Dominguez M, et al. Acute kidney injury is an early predictor of mortality for patients with alcoholic hepatitis. Clin Gastroenterol Hepatol 2012;10:65-71.e3.

13 Bush K, Kivlahan DR, Mcdonell MB, et al. The audit alcohol consumption questions (audit-c): an effective brief screening test for problem drinking. Arch Intern Med 1998;158:1789.

14 Hodgson R, Alwyn T, John B, et al. The fast alcohol screening test. Alcohol Alcohol 2002;37:61-6.

15 National Institute for Healthcare and Clinical Excellence. Alcohol use disorders: diagnosis, assessment, and management of harmful drinking and alcohol dependence. 2011.

16 Day E, Best D, Sweeting R, et al. Detecting lifetime alcohol problems in individuals referred for liver transplantation for nonalcoholic liver failure. Liver Transpl 2008;14:1609-13.

17 Mueller S, Millonig G, Sarovska L, et al. Increased liver stiffness in alcoholic liver disease: differentiating fibrosis from steatohepatitis. World J Gastroenterol 2010;16:966.

18 Lafferty H, Stanley AJ, Forrest EH. The management of alcoholic hepatitis: a prospective comparison of scoring systems. Aliment Pharmacol Ther 2013;38:603-10.

19 Forrest EH, Morris AJ, Stewart S, et al. The Glasgow Alcoholic hepatitis score identifies patients who may benefit from corticosteroids. Gut 2007;56:1743-6.

20 Verma S, Ajudia K, Mendler M, et al. Prevalence of septic events, type 1 hepatorenal syndrome, and mortality in severe alcoholic hepatitis and utility of discriminant function and meld score in predicting these adverse events. Dig Dis Sci 2006;51:1637-43. 
21 Katoonizadeh A, Laleman W, Verslype C, et al. Early features of acute-on-chronic alcoholic liver failure: a Prospective cohort study. Gut 2010;59:1561-9.

22 Louvet A, Wartel F, Castel H, et al. Infection in patients with severe alcoholic hepatitis treated with steroids: Early response to therapy is the key factor. Gastroenterology 2009;137:541-8.

23 Parker R, Armstrong MJ, Corbett C, et al. Systematic review: pentoxifylline for the treatment of severe alcoholic hepatitis. Aliment Pharmacol Ther 2013;37:845-54.

24 Cabre E, Rodriguez-Iglesias P, Caballeria J, et al. Short- and long-term outcome of severe alcohol-induced hepatitis treated with steroids or enteral nutrition: a multicenter randomized trial. Hepatology 2000;32:36-42.

25 Phillips M, Curtis H, Portmann B, et al. Antioxidants versus corticosteroids in the treatment of severe alcoholic hepatitis-a randomised clinical trial. J Hepatol 2006;44:784-90.

26 Moreno C, Langlet P, Hittelet A, et al. Enteral nutrition with or without n-acetylcysteine in the treatment of severe acute alcoholic hepatitis: a randomized multicenter controlled trial. J Hepatol 2010;53:1117-23.

27 Nguyen-Khac E, Thevenot T, Piquet M-A, et al. Glucocorticoids pus N-acetylcysteine in severe alcoholic hepatitis. N Engl J Med 2011;365:1781-9.
28 Mathurin P, Moreno C, Samuel D, et al. Early liver transplantation for severe alcoholic hepatitis. N Engl J Med 2011;365:1790-800.

29 Forrest E, Mellor J, Stanton L, et al. Steroids or pentoxifylline for alcoholic hepatitis (STOPAH): study protocol for a randomised controlled trial. Trials 2013;14. doi:10.1186/1745-6215-14-262

30 Kendrick SF, Henderson E, Palmer J, et al. Theophylline improves steroid sensitivity in acute alcoholic hepatitis. Hepatology 2010;52:126-31.

31 Di Mambro AJ, Parker R, Mccune A, et al. In vitro steroid resistance correlates with outcome in severe alcoholic hepatitis. Hepatology 2011;53:1316-22.

32 Assess Safety and Efficacy of ELAD (extracorporeal Liver Assist System) in Subjects With Alcohol-induced Liver Failure Clincial Trial Identifier NCT01471028. http://www.clinicaltrials.gov (accessed 27 Aug 2013).

33 Morris JM, Dickson S, Neilson M, et al. Granulocytapheresis in the treatment of severe alcoholic hepatitis: a case series. Eur J Gastroenterol Hepatol 2010;22:457-60.

34 Spahr L, Chalandon Y, Terraz S, et al. Autologous bone marrow mononuclear cell transplantation in patients with decompensated alcoholic liver disease: a randomized controlled trial. PloS One 2013;8:e53719. 